\title{
Impacto da síndrome pré-menstrual no estado de humor de atletas
}

\author{
Impact of premenstrual syndrome in the mood state of athletes \\ Lenamar Fiorese Vieira', Patrícia Aparecida Gaion
}

\section{RESUMO}

Introdução: Têm sido relatadas alterações negativas no estado de humor em algumas mulheres durante o ciclo menstrual, sobretudo naquelas que apresentam síndrome prémenstrual (SPM). No entanto, existe uma lacuna na literatura a respeito das alterações no estado de humor durante o ciclo menstrual em atletas Com SPM. Objetivo: Investigar o impacto da SPM no estado de humor de atletas ao longo do ciclo menstrual. Métodos: Fizeram parte da amostra 57 atletas de diferentes esportes, de uma cidade do noroeste do Paraná. Utilizou-se um diário de sintomas, baseado nos critérios do American College of Obstetricians and Gynecologist (ACOG), 2000, e o questionário Profile of Mood States (POMS). As atletas utilizaram o diário e responderam ao questionário ao longo de dois ciclos menstruais. Para análise dos dados foram usados os testes de Shapiro Wilk, de Friedman, de Wilcoxon e o teste $U$ de Mann-Whitney, adotando-se $P<0,05$. Resultados: Verificou-se que apenas atletas com SPM apresentaram aumento na alteração total de humor da última semana para o último dia, sendo a diferença significativa no primeiro ciclo menstrual $(p=0,019)$. Conclusão: A presença de SPM pode afetar o estado de humor de atletas, gerando alterações negativas no período pré-menstrual, especialmente no último dia antes da menstruação.

\section{Palavras-chave \\ Humor, emoções, esportes.}

\section{ABSTRACT}

Introduction: Negative alterations in mood states have been related to some women through the menstrual cycle, mainly on those who show premenstrual syndrome (PMS). However, there is a gap in the literature about the alterations in the mood states during the menstrual cycle in athletes with PMS. Objective: Investigate the impact of PMS in the mood state of athletes through the menstrual cycle. Methods: Were part of the sample 57 athletes from different sports, from a northeast city of Paraná. As instruments were used a Symptoms Dairy, based on the American College of Obstetricians and Gynecologist criteria (ACOG), 2000, and the Profile of Mood States (POMS) questionnaire. The athletes answered the instruments through two menstrual cycles. For the data analysis were used the Shapiro-Wilk test, Friedman test, Wilcoxon test and Mann-Whi-

1 Universidade Estadual de Maringá (UEM), Universidade Estadual de Londrina (UEL), Departamento de Educação Física, Programa de Pós-Graduação Associado em Educação Física. 


\section{Keywords}

Mood, emotions, sports. they test, adopting $P<0,05$. Results: It was verified that only athletes with PMS showed a raise in total humor alteration from the last week to the last day, with a significant difference on the 10 menstrual cycle $(p=0,019)$. Conclusion: The presence of PMS can affect the athletes' mood state, generating negative alterations in the pre-menstrual period, especially in the last day of menstruation.

\section{INTRODUÇÃO}

O humor pode ser definido como um estado emocional de duração variável, que transita entre dois polos - um eufórico e outro apático - e afeta diretamente a percepção das experiências reais do indivíduo, ampliando ou reduzindo o impacto delas ${ }^{12}$.

Pessoas com alterações negativas no estado de humor como, por exemplo, humor deprimido podem apresentar dificuldade na mobilização de recursos pessoais, acreditando que suas capacidades não são suficientes para alcançar seus objetivos e, por isso, frequentemente apresentam comportamentos de desistência ou fuga ${ }^{3}$.

Por causa da efemeridade do estado de humor, muitos fatores do indivíduo ou do ambiente podem afetá-lo. No caso das mulheres, o ciclo menstrual desempenha papel importante nas alterações de humor'. Ainda não se tem claro quais as possíveis causas das alterações de humor ao longo do ciclo menstrual, mas acredita-se que alterações hormonais desencadeadas em ciclos menstruais ovulatórios podem provocar uma desregulação serotoninérgica, gerando reduções na serotonina, sobretudo no período pré-menstrual ${ }^{5}$.

Estudos têm indicado que os esteroides sexuais, como o estrogênio e a progesterona, influenciam diretamente no estado de humor e na cognição. O estrogênio apresenta ação antidepressiva e consequente melhora do humor, enquanto a progesterona está associada à diminuição da serotonina, produzindo efeito depressivo ${ }^{6}$.

Ainda que todas as mulheres com ciclos menstruais ovulatórios apresentem alterações hormonais semelhantes, nem todas apresentam alterações negativas de humor no período pré-menstrual', sugerindo que outros fatores, como características psicológicas, podem estar atrelados ${ }^{8}$.

A síndrome pré-menstrual (SPM) é um conjunto de sintomas emocionais e físicos que ocorrem exclusivamente na fase pré-menstrual e restringem significativamente as atividades diárias das mulheres. Muitos sintomas pré-menstruais têm sido relatados, entre os quais podem ser citados sintomas emocionais como depressão, ansiedade, irritabilidade, confusão, explosão de raiva e isolamento e sintomas físicos como mastalgia, desconforto abdominal, cefaleia e edema ${ }^{9}$.
Considerada um distúrbio altamente prevalente entre as mulheres em idade fértil, a SPM, em sua versão mais grave (disforia pré-menstrual), tem sido relacionada à presença de transtornos de humor, especialmente depressão ${ }^{10}$, o que, somado aos outros sintomas da SPM, podem gerar impacto negativo nas diversas atividades diárias das mulheres"1.

Muitos estudos investigaram a relação entre estado de humor e desempenho esportivo ${ }^{12,13}$ e também entre ciclo menstrual e estado de humor de atletas ${ }^{14}$. No entanto, existe uma lacuna na literatura a respeito do impacto da SPM nas alterações de estado de humor de atletas.

Sabe-se que treinamentos intensivos podem gerar impacto negativo no estado de humor de atletas, como aumento da fadiga e diminuição do afeto positivo, indicando proximidade com depressão ${ }^{15}$. Assim, é possível que mulheres atletas sofram maiores alterações de humor, sobretudo no período pré-menstrual.

Diante da importância do estado de humor para o bemestar das atletas e, consequentemente, para o desempenho esportivo ${ }^{12}$, realizou-se um estudo cujo objetivo foi investigar o impacto da SPM no estado de humor de atletas ao longo do ciclo menstrual.

\section{MÉTODOS}

\section{Sujeitos}

Fizeram parte do estudo todas as atletas entre 18 e 49 anos de idade que estavam treinando em uma cidade do noroeste do Paraná, no segundo semestre do ano de 2007, para competições estaduais e nacionais, totalizando 57 atletas de diversas modalidades esportivas.

Como critérios de exclusão foram estabelecidos: a) não possuir ciclo menstrual eumenorreico, com intervalos menores de 21 dias ou maiores que 35 dias $^{16}$; b) ter tido a menarca há menos de dois anos; c) ter usado no período da coleta de dados, ou ter nos três ciclos menstruais antecedentes, anticoncepcionais hormonais, terapias farmacológicas, drogas ilícitas e/ou bebidas alcoólicas em excesso"; d) ter parado de treinar durante o período da coleta de dados; e) não ter respondido aos instrumentos.

Não foi possível diagnosticar transtornos de humor como depressão maior, depressão bipolar ou transtornos 
de ansiedade; por isso, pode ter ocorrido de alguma atleta com essas comorbidades ter sido incluída no estudo. No entanto, caso isso tenha ocorrido, possivelmente essa atleta não esteja no grupo diagnosticado com SPM. Para estar nesse grupo, a atleta deveria apresentar sintomas como depressão ou ansiedade apenas no período pré-menstrual e, sabe-se, tais transtornos, ainda que possam tornar-se mais intensos antes da menstruação ${ }^{17}$, manifestam-se em diferentes períodos da vida do indivíduo, independentemente do ciclo menstrual18,19.

Além disso, é importante destacar que no estudo de Peluso ${ }^{15} \mathrm{com}$ atletas e vestibulandos não foram encontrados indícios de que fatores de vulnerabilidade ligados a sintomatologia psiquiátrica prévia ou afeto negativo de traço tenham influenciado a presença e a intensidade de alterações no estado de humor de atletas, fato que ocorreu de forma diferente com os vestibulandos.

Das 57 atletas acompanhadas, oito foram excluídas por não possuir ciclo menstrual eumenorreico, 12, por usar anticoncepcional hormonal, seis, por parar de treinar durante a coleta de dados e seis, por não apresentar disponibilidade para responder aos diários durante dois ciclos menstruais consecutivos. Assim, a amostra final foi composta por 25 atletas de dez modalidades esportivas - atletismo, basquetebol, ciclismo, ginástica rítmica, handebol, karatê, natação, voleibol, vôlei de praia e xadrez.

\section{Instrumentos}

Para diagnóstico de SPM, utilizou-se um diário de sintomas baseado nos critérios estabelecidos pelo American College of Obstetricians and Gynecologist ${ }^{9}$, o qual continha sintomas emocionais (depressão, ansiedade, confusão, irritabilidade, isolamento e explosão de raiva), sintomas físicos (mastalgia, desconforto abdominal, cefaleia e edema) e uma opção para a atleta relatar algum sintoma que não estivesse contemplado no diário.

O perfil de estado de humor foi avaliado por meio do questionário Profile of Mood States ${ }^{20}$, validado para língua portuguesa por Peluso ${ }^{15}$. É um instrumento autoaplicável, com 65 adjetivos que descrevem sentimentos, aos quais o sujeito atribui um número correspondente a uma escala do tipo Likert, que vai de zero (de jeito nenhum) a quatro (extremamente) e que possibilita a análise de seis dimensões do humor: tensão, depressão, raiva, vigor, fadiga e confusão. A alteração total de humor foi calculada somando-se os valores de tensão, depressão, raiva, fadiga e confusão e subtraindo-se o valor do vigor. Assim, quanto maior é o resultado da alteração total de humor da atleta, pior está seu estado emocional naquele período.

\section{Procedimentos}

Este estudo foi aprovado pelo Comitê de Ética da Universidade onde foi realizado (n 064/2007). Após a assinatura do termo de consentimento livre e esclarecido, as atletas receberam o diário de sintomas e deveriam preenchê-lo a partir do primeiro dia da próxima menstruação. Ao final de cada dia, as atletas deveriam assinalar somente os sintomas que estivessem presentes no seu dia e que tivessem interferido acentuadamente em suas atividades diárias. As atletas responderam ao diário por dois ciclos menstruais consecutivos, e a troca dos diários ocorreu semanalmente pela pesquisadora nos locais de treino.

Foi diagnosticada SPM quando a atleta apresentou a combinação de pelo menos um sintoma emocional e um sintoma físico, ocorridos necessariamente no período prémenstrual (cinco dias antecedentes à menstruação) e ausentes no período folicular (sexto ao décimo dia do $\left(\mathrm{CM}\right.$ ) ${ }^{9}$. A análise dos diários foi feita por um médico ginecologista.

A aplicação do POMS foi feita em cinco momentos durante o ciclo menstrual: $1{ }^{\circ}$ dia do ciclo menstrual (10 dia de menstruação), 70 dia do ciclo menstrual, 140 dia do ciclo menstrual, última semana do ciclo menstrual (um dia na semana que antecedeu a próxima menstruação - semana pré-menstrual) e último dia do ciclo menstrual (dia anterior à próxima menstruação). As atletas foram instruídas a responder o POMS sempre no final do dia, quando recordariam como se sentiram na maior parte do tempo do dia analisado. Para o último dia, o preenchimento foi feito de forma retrospectiva no dia da menstruação, tendo em vista a dificuldade de saber previamente qual seria o último dia do ciclo menstrual. A aplicação do POMS foi feita nos dois ciclos menstruais acompanhados.

Tendo em vista que nem todas as atletas apresentaram SPM nos dois ciclos menstruais acompanhados, as análises foram feitas para cada um dos ciclos menstruais. Assim, no primeiro ciclo menstrual, oito atletas apresentaram SPM e, no segundo ciclo menstrual, sete atletas apresentaram SPM.

\section{Análise estatística}

Para análise dos dados, utilizou-se o teste de Shapiro-Wilk para verificar a normalidade dos dados. Como não houve distribuição normal, utilizaram-se mediana (Me) e amplitude interquartílica $(\mathrm{aQ})$ para caracterizar os grupos e o teste de Friedman para comparações da alteração total de humor ao longo dos ciclos menstruais de cada grupo, seguido do teste de Wilcoxon para identificar onde ocorreram as diferenças significativas no grupo. Para comparações entre os grupos, utilizou-se o teste $U$ de Mann-Whitney. A significância adotada foi de $P<0,05$.

\section{RESULTADOS}

As atletas apresentaram $\mathrm{Me}=21$ (6) anos de idade, $\mathrm{Me}=13$ (2) anos de idade de menarca, $M e=9$ (6) anos de prática do esporte e Me $=12$ (8) horas de treinamento semanal. Três 
atletas (12\%) já haviam disputado competições internacionais, 19 atletas (76\%), competições nacionais e 3 atletas (12\%) haviam competido em nível estadual. No ano da coleta, 2007, 13 atletas (52\%) iriam disputar competições cujo nível mais alto seria estadual e 12 atletas (48\%), competições nacionais.

Quando se analisaram as alterações no estado de humor das atletas sem levar em consideração a presença de SPM, os valores encontrados no 10 ciclo menstrual foram: $\mathrm{Me}=22,5$ (41) para o $1^{\circ} \mathrm{dia}, \mathrm{Me}=4$ (32) para o $70 \mathrm{dia}, \mathrm{Me}=6,5$ $(3,5)$ para o $14^{\circ} \mathrm{dia}, \mathrm{Me}=2,5(25)$ para a última semana e $\mathrm{Me}$ = 8 (38) para o último dia. Já no $2^{\circ}$ ciclo menstrual verificaram-se Me $=11$ (39) para o $1^{\circ} \mathrm{dia}, \mathrm{Me}=10,5$ (38) para o $70 \mathrm{dia}$, $M e=2,5$ (33) para o $14^{\circ} \mathrm{dia}, \mathrm{Me}=8,5$ (36) para a última se- mana e Me = 8,5 (35) para o último dia. Não foram observadas diferenças significativas no total de alteração do humor das atletas em nenhum dos ciclos menstruais analisados (10 ciclo menstrual: $p=0,164 ; 2^{\circ}$ ciclo menstrual: $\left.p=0,835\right)$. No entanto, quando a análise foi feita considerando a presença de SPM, notou-se diferença significativa entre os grupos no último dia do $1{ }^{\circ}$ ciclo menstrual $(p=0,019)$ (Figura 1).

No segundo ciclo menstrual não foram notadas diferenças significativas entre os grupos, embora as atletas com SPM tenham apresentado, em ambos os ciclos menstruais reduções na alteração total de humor do sétimo dia à última semana e aumento da última semana para o último dia (Figura 2).

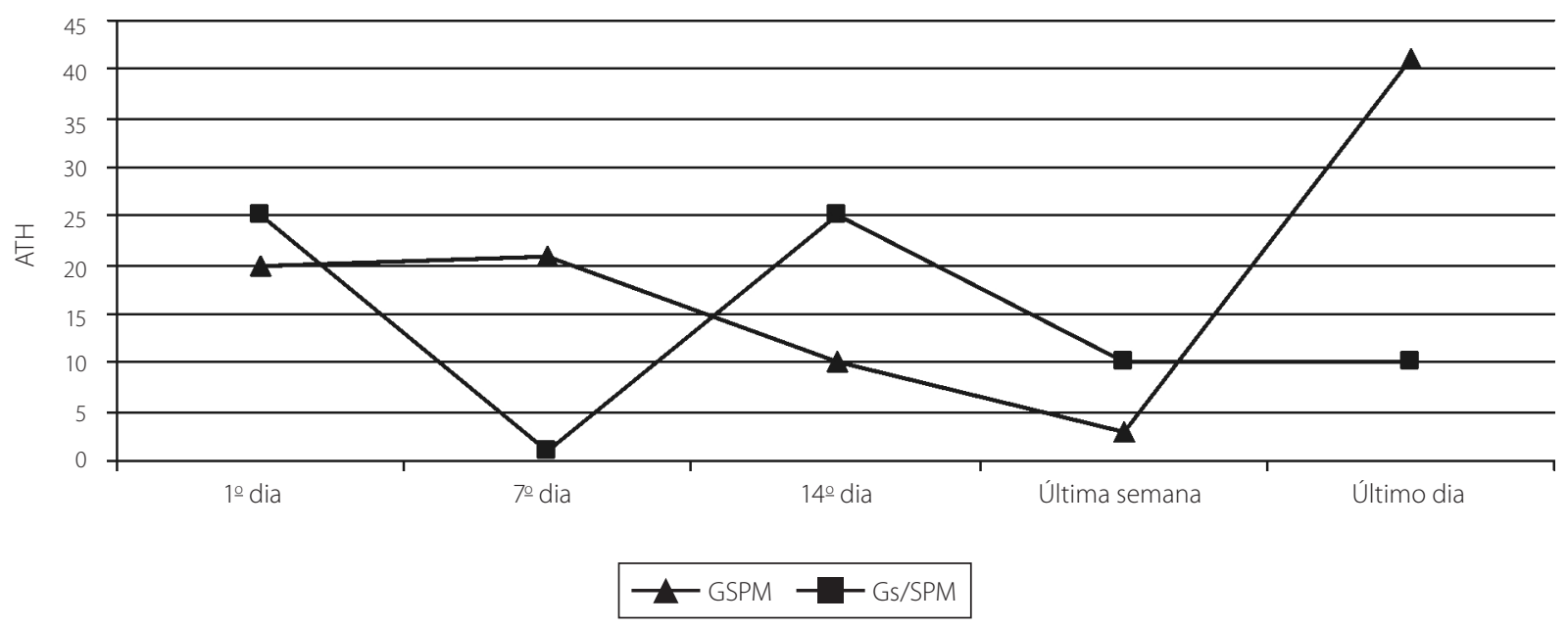

ATH: alteração total de humor; GSPM: grupo com síndrome pré-menstrual; Gs/SPM: grupo sem síndrome pré-menstrual.

Figura 1. Valores de mediana do total de alteração do humor das atletas com $(n=8)$ e sem síndrome pré-menstrual $(n=17)$ ao longo do $1^{\circ}$ ciclo menstrual (diferença significativa no último dia do ciclo menstrual entre grupos com e sem SPM: p = 0,019, segundo o teste $U$ de Mann-Whitney.
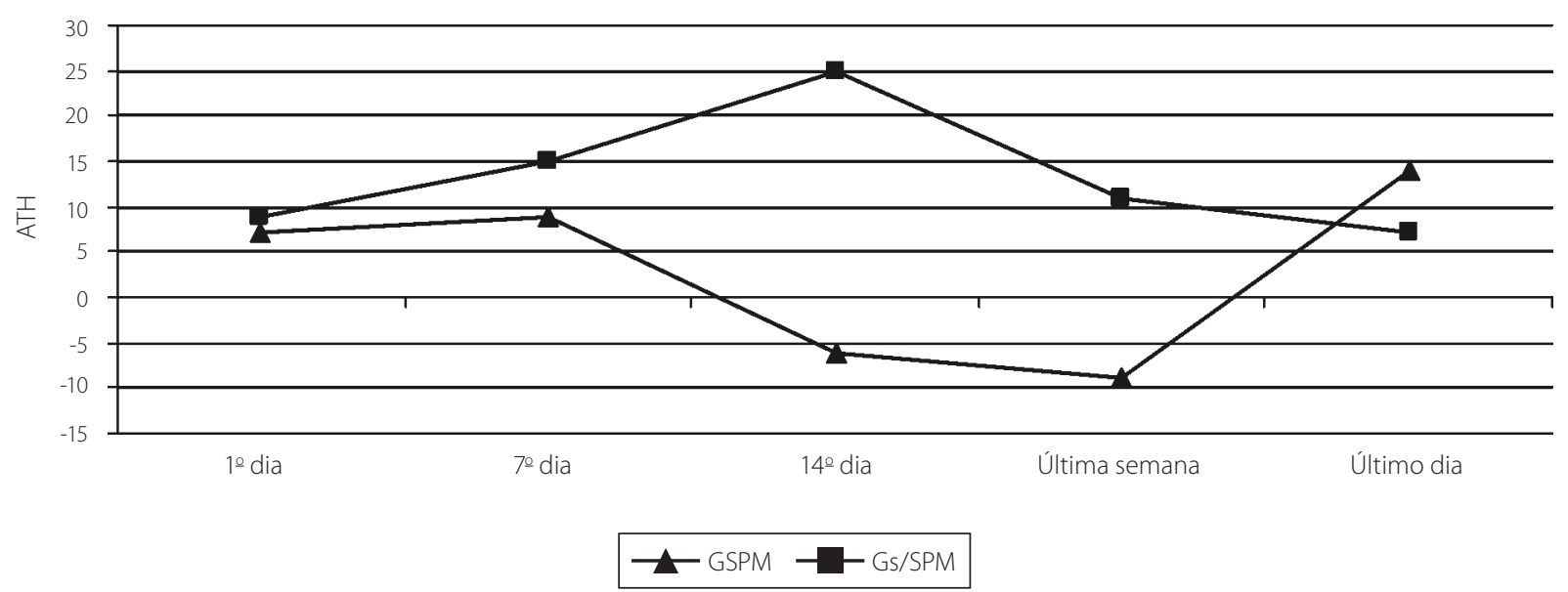

ATH: alteração total de humor; GSPM: grupo com síndrome pré-menstrual; GS/SPM: grupo sem síndrome pré-menstrual.

Figura 2. Valores de mediana do total de alteração do humor das atletas com $(n=7)$ e sem síndrome pré-menstrual ( $n=18)$ ao longo do $2^{\circ}$ ciclo menstrual. 


\section{DISCUSSÃO}

Para conhecimento, este é o primeiro estudo brasileiro que investiga as alterações no estado de humor ao longo do ciclo menstrual em atletas por causa da presença de SPM. Assim como em pesquisas desenvolvidas com diversas populações ${ }^{21-23}$, notou-se que a SPM pode desempenhar alterações afetivas que geram impacto significativo no estado de humor também em populações de atletas. Isso porque, quando se analisou o estado de humor sem levar em consideração a presença de SPM, não foram notadas diferenças significativas no estado de humor ao longo dos ciclos menstruais. No entanto, ao se analisar o estado de humor das atletas com SPM, foram verificados aumentos na alteração total de humor da última semana para o último dia.

O fato de a diferença na alteração total de humor da última semana para o último dia ter sido significativa apenas no primeiro ciclo menstrual pode ser em virtude da percepção da intensidade dos sintomas por parte das atletas. Sabe-se que diversos fatores podem afetar a percepção das pessoas a respeito dos sintomas que acreditam possuir ${ }^{7,24}$, por isso é possível que alguns fatores possam ter influenciado a percepção da intensidade dos sintomas por parte de algumas atletas e feito com que esses sintomas tenham sido percebidos de forma mais intensa no primeiro ciclo menstrual, ocasionando, assim, a diferença estatisticamente significativa apenas nesse ciclo.

Mas é importante frisar que, apesar de a diferença ter sido estatisticamente significativa apenas no primeiro ciclo menstrual acompanhado, houve um padrão característico de diminuição da alteração total de humor do sétimo dia do ciclo à última semana, com aumento da última semana para o último dia em ambos os ciclos menstruais das atletas com SPM.

Esses achados vêm ao encontro da literatura que demonstra que mulheres não atletas com SPM apresentam alterações negativas no humor anteriormente à menstruação, em períodos de uma a duas semanas ${ }^{17,25,26}$. No caso das atletas com SPM do presente estudo, a duração dos sintomas durante o período pré-menstrual apresentou-se em um período menor do que o observado nos estudos citados, uma vez que a menor alteração total de humor foi encontrada até uma semana antes da menstruação.

Já a diferença significativa na alteração total de humor apenas da última semana para o último dia ocorreu possivelmente porque os sintomas da SPM se agravam com o início dos fenômenos regressivos do corpo lúteo, que ocorre por volta do $24^{\circ}$ ou do $25^{\circ}$ dia do ciclo menstrual27.

Outro ponto importante a ser observado foi o padrão do gráfico do total de alteração do humor das atletas com SPM; notou-se que no 10 e no 70 dia dos ciclos menstruais os valores mantiveram-se semelhantes, com diminuições no $14^{\circ}$ e até uma semana antes (quando apresentaram a menor alteração total de humor) e, finalizando o ciclo menstrual, com aumento no último dia.

Esse perfil de total alteração do humor evidencia as oscilações de humor relacionadas à SPM e relatadas na literatura - os sintomas pré-menstruais (e consequente piora do humor) ocorrem no período pré-menstrual e em algumas mulheres podem permanecer até o final da menstruação (que neste estudo ocorreu por volta do $70 \mathrm{dia}$ ), desaparecendo no período entre o fim da menstruação e poucos dias antes da menstruação (sendo a duração do período de bem-estar diferente entre as mulheres) ${ }^{22}$.

Sabe-se que o estado de humor funciona como um filtro das experiências reais pelas quais passa o indivíduo, ampliando ou reduzindo o impacto delas ${ }^{1,2}$. As atletas do presente estudo que apresentaram SPM podem ter dificuldades durante o período pré-menstrual de manter o bemestar e o controle emocional, tão importantes para o desempenho esportivo ${ }^{28}$.

Apresentar estado de humor positivo antes de competição tem sido associado com ótimo desempenho durante competições ${ }^{12,13}$, por isso valores elevados de alteração total de humor no período pré-menstrual podem fazer com que as atletas com SPM percebam de uma maneira ainda mais negativa situações de dificuldade normais nas competições, deixando-se abater e desistindo facilmente.

Em razão da efemeridade do estado de humor, é possível que variáveis como as cargas de treinamento das atletas 29,30 possam ter influenciado os resultados do presente estudo. Esse fator não pode ser controlado, uma vez que para se identificar a prevalência de SPM foi necessário avaliar atletas de diferentes modalidades esportivas. Além disso, a análise do estado de humor teve de ser feita com base no ciclo menstrual de cada atleta, o que, é óbvio, não coincidiu igualmente nos mesmos períodos de treinamento e competição das atletas. Destaca-se também que as limitações referentes ao número de sujeitos na pesquisa devem-se, sobretudo, ao critério utilizado para diagnóstico de SPM ${ }^{9}, 0$ qual aponta uma série de critérios de exclusão que ocasionaram a diminuição no número de atletas participantes.

\section{CONCLUSÃO}

Assim como em outras populações, a presença de SPM pode afetar o estado de humor de atletas, gerando alterações negativas durante o período pré-menstrual, especialmente no último dia de menstruação. É importante que profissionais da saúde vinculados a equipes esportivas femininas estejam atentos ao ciclo menstrual das atletas, disponibilizando suporte emocional para minimizar o impacto da SPM no estado de humor dessas mulheres. 


\section{AGRADECIMENTOS}

À Coordenação de Aperfeiçoamento de Pessoal de Nível Superior (Capes), pela concessão de bolsa, bem como aos técnicos e às atletas das equipes participantes.

\section{REFERÊNCIAS}

1. Dalgalarrondo P. Psicopatologia e semiologia dos transtornos mentais. Porto Alegre: Artmed Sul; 2000.

2. Gazzaniga MS, Heatherton TF. Ciência psicológica: mente, cérebro e comportamento. Porto Alegre: Artmed; 2005.

3. Lane AM. Relationships between perceptions of performance expectations and mood among distance runners: the moderating effect of depressed mood. J Sci Med Sport. 2001;4(1):116-28.

4. Andrade LHSG, Viana MC, Silveira CM. Epidemiologia dos transtornos psiquiátricos na mulher. Rev Psiq Clin. 2006;33(2):43-54.

5. Rapkin A. A review of treatment of premenstrual syndrome and premenstrual dysphoric disorder. Psychoneuroendocrinology. 2003;28(3):39-53.

6. Silva ACJSR, De Sá MFS. Efeitos dos esteroides sexuais sobre o humor e a cognição. Rev Psiq Clin. 2006;33(2):60-7.

7. Campagne DM, Campagne G. The premenstrual syndrome revisited. Eur J Obstet Gynecol Reprod Biol. 2007;130(1):4-17.

8. Halbreich U. Premenstrual dysphoric disorders: a diversified cluster of vulnerability traits to depression. Acta Psychiatr Scand. 1997;95(3):169-76.

9. ACOG (American College of Obstetricians and Gynecologist). Premenstrual syndrome. ACOG Practice Bulletin; 2000.

10. Cheniaux E. Tratamento da disforia pré-menstrual com antidepressivos: revisão dos ensaios clínicos controlados. J Bras Psiquiatr. 2006;55(2):142-7.

11. Espina N, Fuenzalida A, Urrutia MT. Relación entre rendimiento laboral y síndrome premenstrual. Rev Chil Obstet Ginecol. 2005;70(2):113-8.

12. Covassin T, Pero $S$. The relationship between self-confidence, mood state, and anxiety among collegiate tennis players. J Sport Behav. 2004;27(3):230-42.

13. Vieira LF, Fernandes SL, Vieira JLL, Vissoci JRN. Estado de humor e desempenho motor: um estudo com atletas de voleibol de alto rendimento. Rev Bras Cineantropom Desempenho Hum. 2008;10(1):62-8.
14. Cockerill IM, Nevill AM, Byrne NC. Mood, mileage and the menstrual cycle. Br J Sports Med. $1992 ; 26(3): 145-50$.

15. Peluso MAM. Alterações de humor associadas à atividade física intensa [tese]. São Paulo: Universidade de São Paulo; 2003.

16. Creinin MD, Keverline $S$, Meyn LA. How regular is regular? An analysis of menstrual cycle regularity. Contraception. 2004;70(4):289-92.

17. Valadares GC, Ferreira LV, Correa Filho H, Romano-Silva MA. Transtorno disfórico pré-menstrual. Revisão - conceito, história, epidemiologia e etiologia. Rev Psiq Clin. 2006;33(3):117-23.

18. Neves MCC, Dalgalarrondo P. Transtornos mentais autorreferidos em estudantes universitários. J Bras Psiquiatr. 2007;56(4):237-44.

19. Souza LDM, Silva RS, Godoy RV, Cruzeiro AL, Faria AD, Pinheiro RT, et al. Sintomatologia depresssiva em adolescentes iniciais - estudo de base populacional. J Bras Psiquiatr. 2008;57(4):261-6.

20. McNair DM, Lorr M, Droppleman LF. Revised Manual Profile of Mood States. San Diego, CA: Educational and Industrial Testing Service; 1992.

21. Braverman PK. Premenstrual syndrome and premenstrual dysphoric disorder. J Pediatr Adolesc Gynecol. 2007;20(1):3-12.

22. Indusekhar R, Usman SB, O'Brien S. Psychological aspects of premenstrual syndrome. Best Pract Res Clin Obstet Gynaecol. 2007;21(2):207-20.

23. Milewicz A, Jedrzejuk D. Premenstrual syndrome: from etiology to treatment. Maturitas. 2006:55(1):47-54.

24. Rangé B. Terapia racional-emotivo-comportamental. In: Range B, organizador. Psicoterapias cognitivo-comportamentais: um diálogo com a psiquiatria. 1a ed. Porto Alegre: Artmed; 2001. p. 34-48

25. Dickerson LM, Mazyck PJ, Hunter MH. Premenstrual syndrome. Am Fam Physician. 2003;67(8):1743-52.

26. Halbreich U. The etiology, biology, and evolving pathology of premenstrual syndromes. Psychoneuroendocrinology. 2003;28(3):55-99.

27. Bäckstrõm T. Neuroendocrinology of premenstrual syndrome. Clin Obstet Gynecol. 1992; 35(3):612-28.

28. Skinner N, Brewer N. Adaptive approaches to competition: challenge appraisals and positive emotion. J Sport Exerc Psychol. 2004;26(3):283-305.

29. Bouget M, Rouveix M, Michaux 0, Pequignot J, Filaire E. Relationships among training stress, mood and dehydroepiandrosterone sulphate/cortisol ratio in female cyclists. J Sports Sci. 2006;24(12):1297-302.

30. Rohlfs ICPM, Carvalho T, Rotta TM, Krebs RJ. Aplicação de instrumentos de avaliação de estado de humor na detecção da síndrome do excesso de treinamento. Rev Bras Med Esporte. 2004;10(2):111-6. 